\title{
Interaction Quench in Nonequilibrium Luttinger Liquids
}

\author{
Stéphane Ngo Dinh, ${ }^{1}$ Dmitry A. Bagrets, ${ }^{2}$ and Alexander D. Mirlin ${ }^{1,3,4}$ \\ ${ }^{1}$ Institut für Theorie der Kondensierten Materie and DFG Center for Functional Nanostructures, \\ Karlsruhe Institute of Technology, 76128 Karlsruhe, Germany \\ ${ }^{2}$ Institut für Theoretische Physik, Universität zu Köln, Zülpicher Str. 7r7, 50937 Köln, Germany \\ ${ }^{3}$ Institut für Nanotechnologie, Karlsruhe Institute of Technology, 76021 Karlsruhe, Germany \\ ${ }^{4}$ Petersburg Nuclear Physics Institute, 188300 St. Petersburg, Russia
}

(Dated: September 10, 2018)

\begin{abstract}
We study the relaxation dynamics of a nonequilibrium Luttinger liquid after a sudden interaction switch-on ("quench"), focussing on a double-step initial momentum distribution function. In the framework of the non-equilibrium bosonization, the results are obtained in terms of singular Fredholm determinants that are evaluated numerically and whose asymptotics are found analytically. While the quasi-particle weights decay exponentially with time after the quench, this is not a relaxation into a thermal state, in view of the integrability of the model. The steady-state distribution emerging at infinite times retains two edges which support Luttinger-liquid-like power-law singularities smeared by dephasing. The obtained critical exponents and the dephasing length are found to depend on the initial nonequilibrium state.
\end{abstract}

PACS numbers: 71.10.Pm, 67.85.Lm, 03.75.Ss

\section{INTRODUCTION}

Quantum physics of interacting one-dimensional (1D) systems represents a fascinating research area ${ }^{1}$. Most important experimental realizations include electrons in 1D nanostructures (quantum Hall and topological insulator edges, carbon nanotubes, semiconductor quantum wires), quantum spin chains, and cold atoms (bosons as well as fermions) in optical traps. One of central directions of current research is the physics of nonequilibrium phenomena in these structures.

In the cold atoms context (see Ref. 2 for a review, the most frequently considered nonequilibrium setting is a quantum quench ${ }^{3}$-7: : one explores a quantum evolution of the system after a sudden change of one of the parameters. In particular, one can modify the optical lattice potential confining the atoms. An alternative possibility is to suddenly change the interaction strength by employing a strong dependence of interaction on the magnetic field in the vicinity of Feshbach resonance. It has been recognized that, upon an interaction quench, a Luttinger liquid shows an interesting dynamics and eventually evolves into a non-thermal state characterized by nonequilibrium power-law correlations $8-10$. Subsequent theoretical works explored various generalizations of this problem ${ }^{11} 26$.

On the other hand, in the context of electronic systems, typical nonequilibrium setups are of steady-state character (as obtained by applying bias voltages to some of source electrodes). Recent experiments have addressed nonequilibrium spectroscopy of carbon nanotubes 27 and quantum Hall edge states 28 as well as nonequilibrium edge state interferometry 2936 . On the theory side, one of important recent theoretical advances was a development of the method of nonequilibrium bosonization $37[40$ that permits, in particular, to treat Luttinger liquids with distribution functions of incoming electrons that have multiple Fermi edges. It was shown that this leads to a multiple-branch zero-bias anomaly with exponents and dephasing rates controlled by the nonequilibrium state of the system. Related results have been obtained for the problems of quantum Hall edge state spectroscopy 44145 and Mach-Zehnder interferometry 41443 .

While two types of nonequilibrium Luttinger liquid setups (a temporal interaction quench and a steady-state with multiple Fermi edges originating from applied voltages) are quite different, there is a remarkable similarity between the results. In both cases, one finds non-trivial power-law exponents that are essentially different from the equilibrium ones. In this paper, we show that both kinds of nonequilibrium settings can be treated within a unified framework of the nonequilibrium bosonization. We employ this formalism to explore the dynamics after an interaction quench in an initially nonequilibrium Luttinger liquid. The Fermi-edge exponents and dephasing are controlled by charge fractionalization at temporal (and possibly spatial) boundaries of the interaction region and by the input nonequilibrium distributions.

\section{EQUILIBRIUM QUENCH IN THE LUTTINGER MODEL}

The Luttinger model describes the low-energy physics of interacting 1D fermions. It turns out that lowenergy excitations can be fully captured in terms of bosonic modes. A free 1D fermionic system with right(left-)moving modes $\psi_{+}\left(\psi_{-}\right)$close to the Fermi points can be mapped onto a free $1 \mathrm{D}$ bosonic system with a linear spectrum

$$
\begin{aligned}
H_{0} & =-i v_{F}: \int \mathrm{d} x\left(\psi_{+}^{\dagger} \partial_{x} \psi_{+}-\psi_{-}^{\dagger} \partial_{x} \psi_{-}\right): \\
& =\pi v_{F}: \int \mathrm{d} x\left(\varrho_{+}^{2}+\varrho_{-}^{2}\right):
\end{aligned}
$$


with density operators $\varrho_{ \pm}(x)=: \psi_{ \pm}^{\dagger}(x) \psi_{ \pm}(x)$ : and Fermi velocity $v_{F}$. The fermionic operators can be likewise expressed in terms of bosonic operators

$$
\psi_{\eta}(x) \sim \frac{\eta}{\sqrt{2 \pi a}} e^{i \phi_{\eta}}, \quad \eta= \pm
$$

where the phase operators $\phi_{\eta}$ are related to densities via $\varrho_{\eta}(x)=(\eta / 2 \pi) \partial_{x} \phi_{\eta}(x)$. While counterpropagating density modes are uncoupled for originally free fermions, they are mixed by interaction. The interaction Hamiltonian for short-range interaction is

$$
\begin{aligned}
H_{\text {int }}(t)=\frac{g_{4}(t)}{2}: \int \mathrm{d} x\left(\varrho_{+}^{2}+\varrho_{-}^{2}\right) & \\
& +g_{2}(t): \int \mathrm{d} x \varrho_{+}(x) \varrho_{-}(x): .
\end{aligned}
$$

To describe the interaction quench we let the coupling parameters $g_{j}(t)=g_{j} \theta(t)$ be time dependent. In more physical terms we assume that the switching time is much shorter than all characteristic time scales of the problem set for example by the inverse voltage $U$, see Sec. III A below. In the presence of interaction the new bosonic eigenmodes, the "plasmons" $\tilde{\varrho}_{\eta}$, are obtained by the Bogoliubov transformation

$$
\left(\begin{array}{l}
\varrho_{+} \\
\varrho_{-}
\end{array}\right)=\left(\begin{array}{ll}
c & s \\
s & c
\end{array}\right)\left(\begin{array}{l}
\tilde{\varrho}_{+} \\
\tilde{\varrho}_{-}
\end{array}\right), \quad c \equiv \frac{1+K}{2 \sqrt{K}}, s \equiv \frac{1-K}{2 \sqrt{K}}
$$

with the Luttinger parameter

$$
K=\sqrt{\frac{2 \pi v_{F}+g_{4}-g_{2}}{2 \pi v_{F}+g_{4}+g_{2}}} .
$$

The full Hamiltonian after the quench then reads

$$
H=H_{0}+H_{\text {int }}(t>0)=\pi u: \int \mathrm{d} x\left(\tilde{\varrho}_{+}^{2}+\tilde{\varrho}_{-}^{2}\right):
$$

with the plasmon velocity

$$
u=v_{F} \sqrt{\left(1+g_{4} /\left(2 \pi v_{F}\right)\right)^{2}+\left(g_{2} /\left(2 \pi v_{F}\right)\right)^{2}} .
$$

In thermal equilibrium the many-body density matrix $\hat{\varrho}=\mathcal{Z}^{-1} e^{-H / T}$ is a function of $H$, thus it is straightforwardly expressed as an exponential of bilinears of bosonic fields.

Ref. 814 considered time-evolution after a sudden interaction switch-on. The initially noninteracting system is prepared in the thermal equilibrium state $\hat{\varrho}_{0}=$ $\mathcal{Z}_{0}^{-1} e^{-H_{0} / T}$ which after the quench no longer represents equilibrium (with respect to the full Hamiltonian $H$ ). However, the time-evolution of $\hat{\varrho}_{0}, \varrho_{\eta}$ and $\psi_{\eta}$ can be deduced by the Bogoliubov transformation (1). Calculations there were performed with a finite interaction range $R_{0} \sim v_{F} / \Lambda$ as short-distance regularization. At long distances $\bar{x} \gg R_{0}$ results are insensitive to the regularization scheme, and momentum-dependent coupling parameters (associated with finite interaction range) can be replaced by their zero momentum values, $g_{j}(q) \approx g_{j}(q=0)$. The equal-time correlation function then is

$$
G_{\mathrm{eq}+}^{<}(\bar{x}, \bar{t} ; 0, \bar{t})=G_{0+}^{<}(\bar{x}, 0)\left|\frac{R_{0}}{\bar{x}}\right|^{\tilde{\gamma}^{2}}\left|\frac{(2 u \bar{t})^{2}-\bar{x}^{2}}{(2 u \bar{t})^{2}}\right|^{\tilde{\gamma}^{2} / 2},
$$

where the exponent is determined by $\tilde{\gamma} \equiv\left(1-K^{2}\right) / 4 K$ and $G_{0+}^{<}(\bar{x}, 0)$ is the free fermionic Green's function.

For short times such that $2 u \bar{t} \ll|\bar{x}|$ the correlation function $G_{+}^{<}(\bar{t} ; \bar{x}, 0) \approx Z(\bar{t}) G_{0+}^{<}(\bar{x} ; 0)$ can be interpreted as the Green's function of an effective time-dependent Fermi liquid with "Landau quasiparticle weight"

$$
Z(\bar{t})=\left(R_{0} / 2 u \bar{t}\right)^{\tilde{\gamma}^{2}}
$$

which gives rise to a discontinuity in the momentum distribution function $n_{+}(p)$ at Fermi momentum $p=p_{F}$. According to (4) the jump decays algebraically with time $\bar{t}$.

For large times $\bar{t} \rightarrow \infty$ the system reaches a timeindependent steady state with power-law correlations

$$
G_{+}^{<}(\bar{t} \gg \bar{x} / u ; \bar{x}, 0)=G_{0+}^{<}(0, \bar{x})\left|R_{0} / \bar{x}\right|^{\tilde{\gamma}^{2}} .
$$

The corresponding momentum distribution function no longer exhibits a discontinuity at $p=p_{F}$, but instead has a power-law singularity $\sim\left|p-p_{F}\right|^{\tilde{\gamma}^{2}}$. This behavior is very similar to that observed in an equilibrium Luttinger liquid ${ }^{1}$, however with an exponent $\tilde{\gamma}^{2}$ that differs from the equilibrium one, $2 \gamma=(1-K)^{2} / 2 K$. Hence, while interactions drive the dynamical evolution which destroy the Fermi-liquid character of the spectral function, the integrability prevents the system from relaxation into thermal equilibrium.

\section{QUENCH IN THE OUT-OF-EQUILIBRIUM LUTTINGER MODEL}

In this section we consider the quench problem in the Luttinger liquid prepared in a nonequilibrium initial state with double-step distribution functions. First we present the key details of our calculations within the nonequilibrium bosonization framework and then discuss the obtained results.

\section{A. Solution via nonequilibrium bosonization}

In our paper we consider initial states in which fermionic right-/left-moving single-particle states $\left( \pm, \epsilon_{i}\right)$ are independently occupied according to distribution functions $f_{ \pm}\left(\epsilon_{i}\right)=(1-a) \theta\left(-\epsilon_{i}\right)+a \theta\left(U-\epsilon_{i}\right)$ which have two sharp Fermi edges at $\epsilon=0$ and $U$. For such nonequilibrium states the initial density matrix is not an exponential of an operator quadratic in the plasmonic 


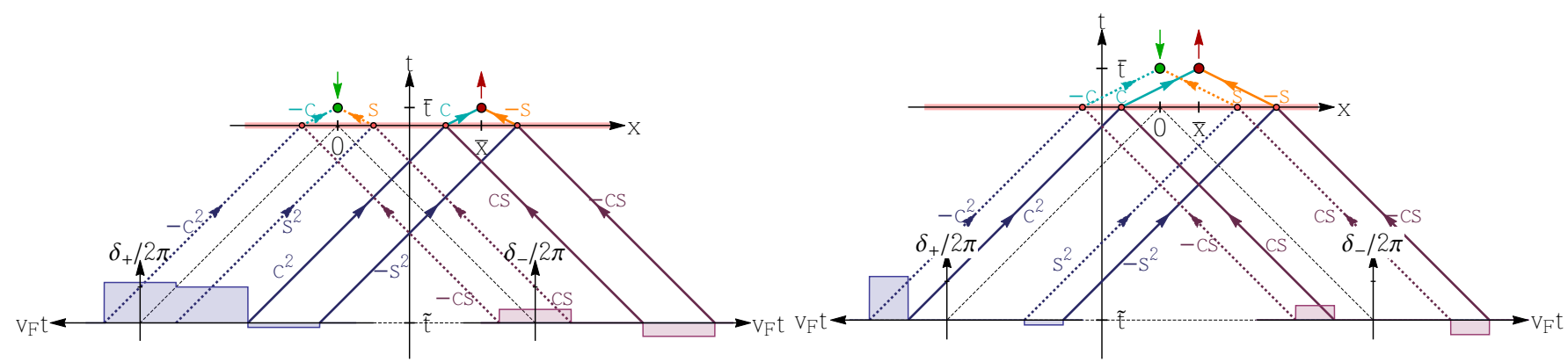

FIG. 1: Trajectories of density peaks and the corresponding counting phases $\delta_{ \pm}$for the Green's function 5 in the case of short times $2 u \bar{t}<\bar{x}$ (left) and long times $2 u \bar{t}>\bar{x}>0$ (right). The $\mathrm{x}$-axis corresponds to the time $t=0$ when the quench takes place.

modes. This makes the nonequilibrium problem considerably more complicated in comparison with the equilibrium one.

A general framework to deal with this class of problems has been developed in Ref. 37 where the operator bosonization method was combined with the Keldysh action technique. Within this approach the right-movers' single-particle Green's function

$$
\begin{aligned}
i G_{+}^{<}(\bar{x}, \bar{t} ; 0, \bar{t}) & \equiv-\left\langle\psi_{+}^{\dagger}(0, \bar{t}) \psi_{+}(\bar{x}, \bar{t})\right\rangle \\
& =i G_{\mathrm{eq}+}^{<}(\bar{x}, \bar{t} ; 0, \bar{t}) \prod_{\eta= \pm} \Delta_{\eta}\left[\delta_{\eta}\right] / \Delta_{0}\left[\delta_{\eta}\right]
\end{aligned}
$$

can be expressed in terms of a functional determinant of a Fredholm operator,

$$
\Delta_{\eta}\left[\delta_{\eta}\right]=\operatorname{Det}\left[\mathbb{1}-f_{\eta}+e^{i \delta_{\eta}} f_{\eta}\right] .
$$

While the distribution function $f_{\eta}$ is diagonal in energy representation, the counting phase

$$
\delta_{\eta}(t)=4 \pi v_{F} \lim _{\tilde{t} \rightarrow-\infty} \int_{\tilde{t}-t}^{0} \mathrm{~d} \tau \varrho_{\eta}^{q}\left(\eta v_{F} \tau, \tilde{t}\right)
$$

is diagonal in the conjugate time representation. The equilibrium value $\Delta_{0}\left[\delta_{\eta}\right]$ of $\Delta_{\eta}\left[\delta_{\eta}\right]$ is obtained by replacing $f_{\eta}$ by the equilibrium distribution function $f_{0}(\epsilon)=$ $\theta(-\epsilon)$. The counting phase is sensitive to the asymptotic behavior of the density trajectory $\varrho_{\eta}^{q}$, which is the advanced solution of the classical equations of motion

$$
\begin{aligned}
& {\left[\partial_{t}+\left(v_{F}+\frac{g_{4}}{2 \pi}\right) \partial_{x}\right] \varrho_{+}^{q}(x, t)+\frac{g_{2}}{2 \pi} \partial_{x} \varrho_{-}^{q}(x, t)=J(x, t),} \\
& {\left[\partial_{t}-\left(v_{F}+\frac{g_{4}}{2 \pi}\right) \partial_{x}\right] \varrho_{-}^{q}(x, t)-\frac{g_{2}}{2 \pi} \partial_{x} \varrho_{+}^{q}(x, t)=0 .}
\end{aligned}
$$

The source term $J(x, t) \equiv \frac{1}{2} \delta(t-\bar{t})[\delta(x)-\delta(x-\bar{x})]$ on the right-hand side of the equation corresponds to the injection of a right-moving fermion at position 0 and time $\bar{t}$ and its removal at $(\bar{x}, \bar{t})$. After the quench, $t>0$, the interaction couples right- and left-moving density modes. To decouple them we apply the Bogoliubov transformation (1), yielding

$$
\begin{aligned}
& {\left[\partial_{t}+u \partial_{x}\right] \tilde{\varrho}_{+}^{q}(x, t)=c J(x, t),} \\
& {\left[\partial_{t}-u \partial_{x}\right] \tilde{\varrho}_{-}^{q}(x, t)=-s J(x, t) .}
\end{aligned}
$$

The charge configuration $\tilde{\varrho}_{\eta}^{q}$ describes the "advanced charge response" to the aforementioned injection and removal of the right-moving fermion. It is a superposition of $\delta$-peaks moving with velocities $\pm u$ after the quench and $\pm v_{F}$ prior to it.

Solving the linear Eqs. (8) we find that at times $t>0$ after the quench

$$
\begin{aligned}
\tilde{\varrho}_{+}^{q}(x, t) & =-\frac{1}{2} c \theta(\bar{t}-t) \\
& \times\{\delta[x-u(t-\bar{t})]-\delta[x-\bar{x}-u(t-\bar{t})]\}, \\
\tilde{\varrho}_{-}^{q}(x, t) & =\frac{1}{2} s \theta(\bar{t}-t) \\
& \times\{\delta[x+u(t-\bar{t})]-\delta[x-\bar{x}+u(t-\bar{t})]\} .
\end{aligned}
$$

Requiring continuity at $t=0$ and inverting the Bogoliubov transformation we obtain the charge density prior to the quench, $t<0$,

$$
\begin{aligned}
\varrho_{+}^{q}(x, t) & =-\frac{1}{2}\left\{c^{2}\left(\delta\left[x+u \bar{t}-v_{F} t\right]-\delta\left[x-\bar{x}+u \bar{t}-v_{F} t\right]\right)\right. \\
& \left.-s^{2}\left(\delta\left[x-u \bar{t}-v_{F} t\right]-\delta\left[x-\bar{x}-u \bar{t}-v_{F} t\right]\right)\right\}, \\
\varrho_{-}^{q}(x, t) & =-\frac{1}{2} c s\left\{\left(\delta\left[x+u \bar{t}+v_{F} t\right]-\delta\left[x-\bar{x}+u \bar{t}+v_{F} t\right]\right)\right. \\
& \left.-\left(\delta\left[x-u \bar{t}+v_{F} t\right]-\delta\left[x-\bar{x}-u \bar{t}+v_{F} t\right]\right)\right\} .
\end{aligned}
$$

The latter yields the counting phases

$$
\begin{aligned}
\delta_{+}(t) & =2 \pi\left\{c^{2}\left(\theta\left[t-\frac{-\bar{x}+u \bar{t}}{v_{F}}\right]-\theta\left[t-\frac{u \bar{t}}{v_{F}}\right]\right)\right. \\
& \left.-s^{2}\left(\theta\left[t-\frac{-\bar{x}-u \bar{t}}{v_{F}}\right]-\theta\left[t-\frac{-u \bar{t}}{v_{F}}\right]\right)\right\}, \\
\delta_{-}(t) & =-2 \pi c s\left\{\left(\theta\left[t-\frac{-u \bar{t}}{v_{F}}\right]-\theta\left[t-\frac{\bar{x}-u \bar{t}}{v_{F}}\right]\right)\right. \\
& \left.-\left(\theta\left[t-\frac{u \bar{t}}{v_{F}}\right]-\theta\left[t-\frac{\bar{x}+u \bar{t}}{v_{F}}\right]\right)\right\} .
\end{aligned}
$$

We visualize the above solutions in Fig. 1 which shows the $\delta$-peak density trajectories in the $(x, t)$-plane. The 
filled circles indicate the fermion injection and removal at time $\bar{t}$. Each fermion fractionalizes into right- and leftmoving plasmonic modes with weights $c$ and $-s$. At the time of quench $(t=0)$ the plasmonic peaks disintegrate into bare particle-hole pair excitations (small circles on $x$-axis) which propagate with smaller velocity $v_{F}$. The counting phases $\delta_{ \pm}$are calculated at $\tilde{t} \rightarrow-\infty$.

The left panel of Fig. 1 depicts the situation of $2 u \bar{t}<$ $|\bar{x}|$ and the right panel corresponds to the case $2 u \bar{t}>|\bar{x}|$. In the second case the phases $\delta_{\eta}$ split into two independent pulses $\delta_{\eta}^{(1)}, \delta_{\eta}^{(2)}$ of duration $|\bar{x}| / v_{F}$ which are shifted by $2 u \bar{t} / v_{F}$. In the long-time limit $2 u \bar{t} \gg|\bar{x}|$, the coherence of right- and left-moving plasmons is negligible and the determinant $\Delta_{\eta}\left[\delta_{\eta}\right] \simeq \Delta_{\eta}\left[\delta_{\eta}^{(1)}\right] \Delta_{\eta}\left[\delta_{\eta}^{(2)}\right]$ factorizes into two single-pulse determinants which are of Toeplitz type.

Following Ref. 38 we regularize the determinants $\Delta_{\eta}\left[\delta_{\eta}\right]$ by introducing an ultraviolet cutoff $\Lambda$ and discretize times in steps $\Delta t=\pi / \Lambda$. For counting phases $\delta_{\eta}$ which are vanishing outside some time interval of length $\tau$, the discretization gives rise to $N \times N$-matrices with $N=\tau \Lambda / \pi$. Here we are interested in $\delta_{\eta}(t)$ which are piecewise constant functions. Such dependence leads to the matrices of the generalized Toeplitz form (see Appendix A). Various mathematical results exist for the long-time asymptotic behavior of their determinants. In the simplest situation that $\delta_{\eta}$ are single rectangular pulses [as e.g. $\left.\delta_{\eta}^{(1)}(t)\right]$ matrices are of Toeplitz form with symbols exhibiting Fisher-Hartwig singularities. The Fisher-Hartwig conjecture ${ }^{46}$ then gives the leading exponential and power-law contribution to $\Delta_{\eta}\left[\delta_{\eta}\right]$ for $N \gg 1$ (including numerical prefactors). The extension proposed in Ref. 38 allows for the calculation of subleading power-law contributions. The phases $\delta_{ \pm}(t)$ shown in
Fig. 1 are not of a simple rectangular form. They stem from the superposition of two rectangular pulses and therefore possess four step-like discontinuities in time. This leads to a class of matrices that are a generalization of Toeplitz matrices. The asymptotic behavior of the corresponding determinants can be found by a further generalization of the Fisher-Hartwig conjecture (see Appendix A) which was put forward in Ref. 39. This conjecture was supported both by analytica ${ }^{\sqrt{39}}$ and numerica ${ }^{39 / 41}$ arguments.

In Sec. IIIB we present and discuss the results for the Green's function (5) obtained by means of the analysis of corresponding singular Fredholm determinants. The details of these calculation can be found in Appendix B.

\section{B. Results}

In general the determinants entering Eq. (5) can be efficiently evaluated numerically 39141 . For sufficiently long times $u \bar{t} \gg v_{F} / U$ analytical asymptotics can be found as we discuss below.

For an arbitrary relation between $\bar{x}$ and $u \bar{t}$ we have found that the Green's function is a linear combination of terms involving different powers of $|\bar{x}|,|2 u \bar{t}+\bar{x}|,|2 u \bar{t}-\bar{x}|$, and $2 u \bar{t}$. For simplicity we will focus on two limiting cases: (i) long-distance behavior at finite times, $\bar{x} \gg 2 u \overline{\text {, }}$, and (ii) the stationary limit $\bar{t} \rightarrow \infty$. We also assume a moderate repulsive interaction.

For finite times $\bar{t}$ and long distances $\bar{x} \gg u \bar{t} \gg v_{F} / U$ the Green's function is determined by two dominant terms,

$$
G_{+}^{<}(\bar{x}, \bar{t} ; 0, \bar{t})=G_{0+}^{<}(\bar{x}, 0) e^{-\bar{t} /\left(2 \tau_{\varphi}\right)}\left[\tilde{\Gamma}_{1}^{\prime}\left(\frac{2 u \bar{t} \Lambda}{\pi v_{F}}\right)^{T_{1}^{\prime}}\left(\frac{\pi U}{\Lambda}\right)^{V_{1}^{\prime}}+\tilde{\Gamma}_{2}^{\prime} e^{i \bar{x} U / v_{F}}\left(\frac{2 u \bar{t} \Lambda}{\pi v_{F}}\right)^{T_{2}^{\prime}}\left(\frac{\pi U}{\Lambda}\right)^{V_{2}^{\prime}}\right]
$$

with exponents

$$
T_{j}^{\prime}\left(V_{j}^{\prime}\right)=-\operatorname{Re}\left[\left(\frac{3}{2}-j+s^{2}+2 \beta\right)^{2}+(s+2 \tilde{\beta})^{2}-\frac{1}{4} \pm 2 c^{2} s^{2}\right], \quad j=1,2
$$

and decay rate

$$
\tau_{\varphi}^{-1}=-4 U \frac{u}{v_{F}} \operatorname{Im}(\beta+\tilde{\beta}),
$$

where

$$
\begin{aligned}
& \beta=\frac{1}{2 \pi i} \ln \left[a e^{-2 \pi i s^{2}}+1-a\right], \\
& \tilde{\beta}=\frac{1}{2 \pi i} \ln \left[a e^{2 \pi i c s}+1-a\right],
\end{aligned}
$$

and $\tilde{\Gamma}_{j}^{\prime}$ are numerical prefactors.
Similar to the equilibrium quench, the entire $\bar{x}$ dependence of the interacting Green's function after the quench is given by the noninteracting factor, $G_{0+}^{>} \propto \bar{x}^{-1}$ so that correlations drop off with distance in a Fermiliquid-like manner. Correspondingly, the momentum distribution function has discontinuities at $p=p_{F}$ and $p=p_{F}+U / v_{F}$, signaling the existence of Landau quasiparticle states (see Fig. III B for $K=0.8$ and $a=0.8$ ). In the nonequilibrium setup each of the two Fermi edges 

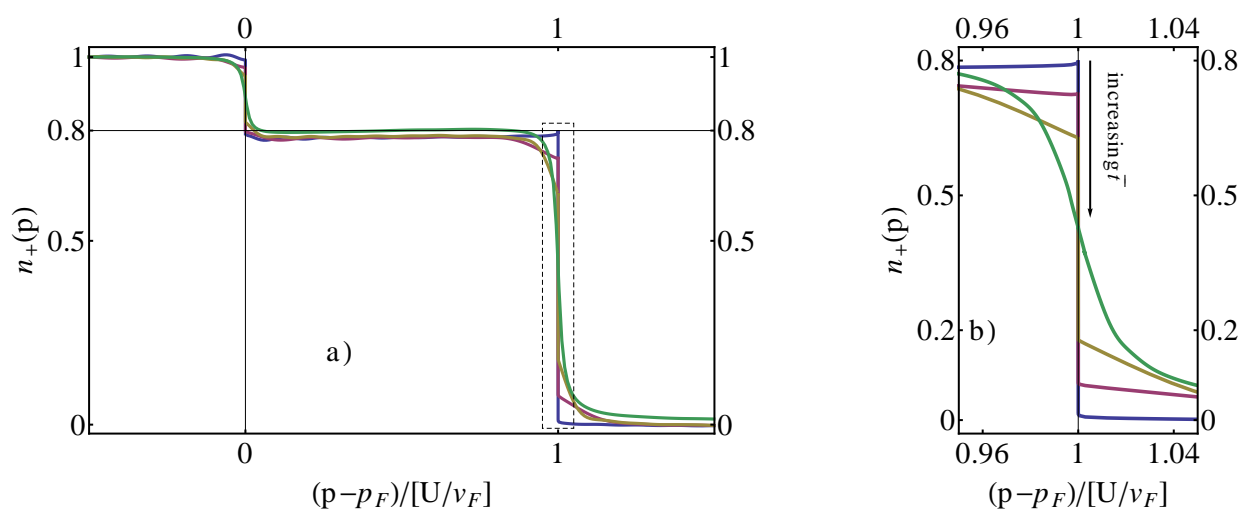

FIG. 2: (a) The momentum distribution function for $K=0.8$ and $a=0.8$ at times $\bar{t} U u / v_{F}=1,10,25$, and in the stationary limit $\bar{t} \rightarrow \infty$, as obtained by a numerical evaluation of the determinants in Eq. (5). Clear discontinuities at the edges $p=p_{F}$ and $p=p_{F}+U / v_{F}$ [see zoom (b)] are visible which decrease with time $\bar{t}$ according to Eq. 15 and eventually vanish.

exhibits quasiparticles with (in general different) weights

$$
Z_{1} \propto e^{-\bar{t} /\left(2 \tau_{\varphi}\right)} \bar{t}^{T_{1}^{\prime}} U^{V_{1}^{\prime}}, \quad Z_{2} \propto e^{-\bar{t} /\left(2 \tau_{\varphi}\right)} \bar{t}^{T_{2}^{\prime}} U^{V_{2}^{\prime}} .
$$

In striking contrast to the equilibrium situation, the quasiparticle weights are not simply algebraically suppressed with time, but also exhibit exponential decay with characteristic time $\tau_{\varphi}$ due to nonequilibrium dephasing.
Let us now turn to the long-time limit, $\bar{t} \rightarrow \infty$. The quenched system then relaxes to a stationary state without Fermi liquid discontinuities, but with critical powerlaw correlations characteristic for Luttinger liquid. In the case of moderate repulsive interaction $\sqrt{2}-1 \leq K \leq 1$ and at $\bar{x} \gg v_{F} U^{-1}$ leading contributions to the Green's function read

$$
G_{+}^{<}(\bar{t} \gg \bar{x} / u ; \bar{x}, 0)=G_{0+}^{<}(0 ; \bar{x}) e^{-\kappa|\bar{x}|}\left(\tilde{\Gamma}_{1}\left|\frac{\Lambda \bar{x}}{\pi v_{F}}\right|^{1+X_{1}}\left|\frac{\pi U}{\Lambda}\right|^{V_{1}}+\tilde{\Gamma}_{2} e^{i U \bar{x} / v_{F}}\left|\frac{\Lambda \bar{x}}{\pi v_{F}}\right|^{1+X_{2}}\left|\frac{\pi U}{\Lambda}\right|^{V_{2}}\right)
$$

with exponents

$$
X_{j}\left(V_{j}\right)=-\frac{1}{2} \operatorname{Re}\left[\left(s^{2}-2 \beta\right)^{2}-\left(s^{2}-2+j+2 \beta^{*}\right)^{2}-(c s-2 \tilde{\beta})^{2}-\left(c s-2 \tilde{\beta}^{*}\right)^{2} \pm\left(c^{2}+s^{2}\right)^{2}\right], \quad j=1,2,
$$

where star denotes the complex conjugation. The decay length $\kappa^{-1}$ is equal to $\kappa^{-1}=4 u \tau_{\varphi}$ with $\tau_{\varphi}$ given by Eq. 12. The numerical prefactors $\tilde{\Gamma}_{j}$ can be found in Appendix B, see Eq. (B1).

Our results show that the limiting (long-time) stationary state retains information about the system's prehistory, i.e. about the initial state prior to the quench. Indeed, at long times after the quench the momentum distribution function exhibits a double-step structure reminiscent of the original nonequilibrium state (see Fig. 33. The initial sharp discontinuities in $n(p)$ are replaced by power laws $\left|p-p_{F}\right|^{q_{1}}$ and $\left|p-p_{F}-U / v_{F}\right|^{q_{2}}$ with $q_{1,2}=-\left(1+X_{1,2}\right)$ which are smeared by nonequilibrium dephasing. The dependence of exponents $q_{1,2}$ and of the inverse dephasing length $\kappa$ on the interaction strength $K$ for a particular choice of the initial two-step distribution $(a=0.8)$ is shown in Figs. 4 and 5.

We stress that the exponents (17) differ from those in the steady-state setup where the Luttinger liquid is coupled to noninteracting reservoirs with nonequilibrium electron distributions 38 . Clearly, they also differ both from the equilibrium exponent ${ }^{1]}$ and from that emerging after a quench in an equilibrium Luttinger liquid, see Sec. II]

\section{SUMMARY}

To summarize, we have studied the dynamics of a nonequilibrium Luttinger liquid after a sudden interaction quench by employing the nonequilibrium bosonization formalism. At variance with a quench out of an 


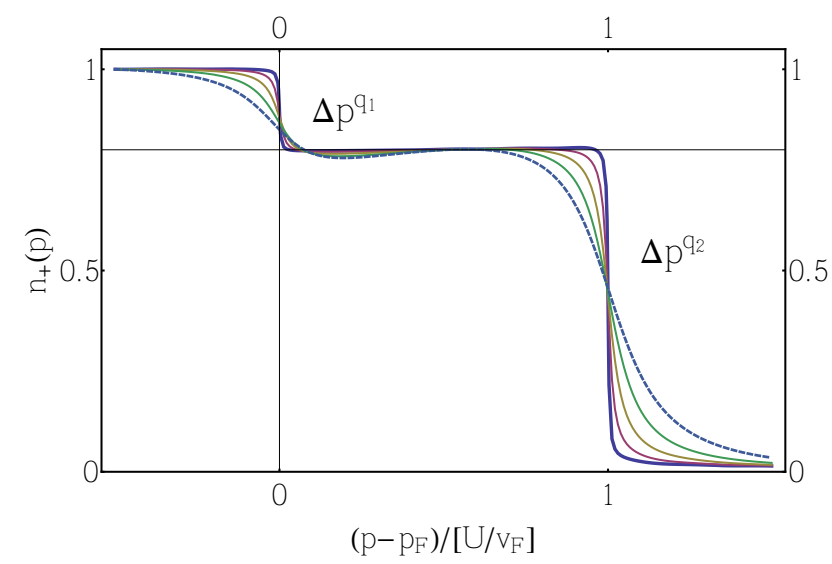

FIG. 3: Steady-state momentum distribution function for $a=$ 0.8 and $K=0.9$ (solid blue line), 0.8, 0.7, 0.6, 0.5 (dashed blue line) obtained by a numerical evaluation of the determinants in Eq. (5). At edges $p=p_{F}$ and $p=p_{F}+U / v_{F}$ the initial discontinuities are replaced by power laws $\Delta p^{q_{1,2}}$ smeared by dephasing.

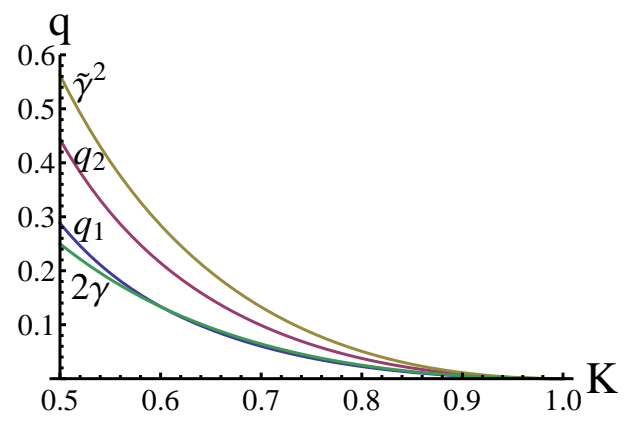

FIG. 4: Luttinger liquid exponents governing power-law singularities of the momentum distribution functions: $q_{1}$ at $p=p_{F}$ and $q_{2}$ at $p=p_{F}+U / v_{F}$ for quenched nonequilibrium with $a=0.8, \tilde{\gamma}^{2}$ for quenched equilibrium, and $2 \gamma$ for equilibrium setup.

initial equilibrium state ${ }^{8114}$, the quasiparticle weights decay exponentially with time after the quench. This exponential behavior is however not a relaxation into a thermal state, which is related to the integrability of the model. In particular, for an initial distribution with two Fermi edges, the distribution emerging at long times retains a double-step structure, with power-law singularities smeared by dephasing. The corresponding exponents as well as the dephasing rate depend on the initial nonequilibrium state.

In conclusion we mention that the framework developed here is also applicable to a more general situation when the interaction region possesses both spatial and temporal boundaries. In this case the counting phases will be determined by the fractionalization processes on all the boundaries.

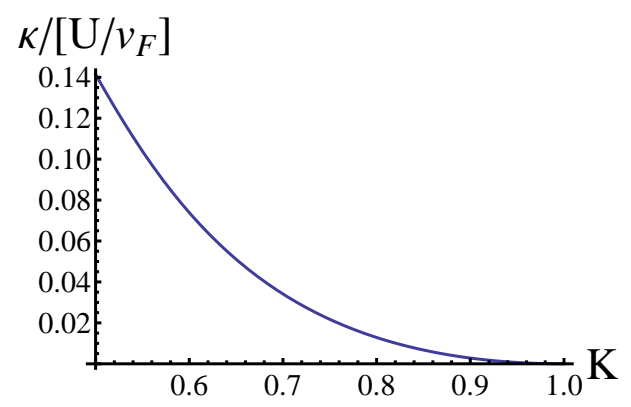

FIG. 5: Inverse decay length $\kappa$ in units of $U / v_{F}$ as a function of the interaction constant $K$ shown for the double-step distribution with $a=0.8$.

\section{ACKNOWLEDGEMENT}

A.D.M. thanks M.A. Cazalilla for an instructive discussion. This work was supported by the collaborative research grant SFB/TR12 of the DFG and by GermanIsraeli Foundation. D.B. is grateful to the TKM institute at KIT for its hospitality.

\section{Appendix A: Generalized Toeplitz determinants}

In this appendix we summarize the main results of Ref. 39 for the asymptotic behavior of generalized Toeplitz determinants. We consider a generalized Toeplitz matrix

$$
g_{j, k}=\int_{-\Lambda}^{\Lambda} \frac{d \epsilon}{2 \Lambda} e^{-i \epsilon \pi / \Lambda\left[j-k-\delta\left(t_{j}\right) /(2 \pi)\right]} \tilde{g}\left(t_{j}, \epsilon\right)
$$

which is defined via its symbol

$$
\tilde{g}(t, \epsilon) \equiv 1+\left(e^{i \delta(t)}-1\right) f(\epsilon) .
$$

Let us focus on the special case when both the phase $\delta(t)$ and the distribution function $f(\epsilon)$ are piecewise constant functions with jumps at times $\tau_{1}<\tau_{2}<\ldots<\tau_{N_{\tau}}$ and energies $\mu_{1}<\mu_{2}<\ldots<\mu_{N_{\mu}}$, respectively. They satisfy the boundary conditions $\delta(t)=0$ for $t \notin\left[\tau_{1}, \tau_{N_{\tau}}\right]$, $f(\epsilon)=1$ for $\epsilon<\mu_{1}$, and $f(\epsilon)=0$ for $\epsilon>\mu_{N_{\mu}}$. The discontinuity points define a grid which subdivides the time-energy plane in domains with different values of the symbol. The domains can be labeled by the time indices $j \in\left\{0, \ldots, N_{\tau}\right\}$, and energy indices $k \in\left\{0, \ldots, N_{\mu}\right\}$. One associates with this set of domains a set of number $c_{j k}$,

$$
\begin{aligned}
c_{j k} & =\frac{1}{2 \pi i} \ln \tilde{g}\left(\tau_{j}+0, \mu_{k}+0\right)+n_{j k}, \\
c_{j 0} & =\delta\left(t_{j}+0\right) /(2 \pi), \quad c_{0 k}=c_{N_{\tau}, k}=c_{j, N_{\mu}}=0 .
\end{aligned}
$$

where $\left\{n_{j k}\right\}$ is an arbitrary set of integers. In the above equation the logarithm $\ln \tilde{g}$ is understood as evaluated 
at its principal branch, $\operatorname{Im} \ln \tilde{g} \in(-\pi, \pi]$. The summation over integers $n_{j k}$ hence amounts to summing over different branches of the logarithms.
It was conjectured in Ref. 39 that the asymptotic behavior of the (normalized) determinant $\bar{\Delta}[\delta(t), f(\epsilon)]=$ $\Delta[\delta(t), f(\epsilon)] / \Delta[\delta(t), T=0]$ takes the form

$$
\bar{\Delta}[\delta(t), f(\epsilon)]=\sum_{\left\{n_{j k}\right\}} \bar{\Gamma}_{\left\{n_{j k}\right\}} \exp \left[i \sum_{1 \leq j \leq N_{t}} \sum_{1 \leq k \leq N_{\mu}} \tau_{j} \beta_{j k} \mu_{k}\right] \prod_{1 \leq j<l \leq N_{t}} \prod_{1 \leq k<m \leq N_{\mu}}\left[\left(\tau_{l}-\tau_{j}\right)\left(\mu_{k}-\mu_{m}\right)\right]^{\gamma_{j l, k m}},
$$

where

$$
\gamma_{j l, k m}=-c_{j k} c_{l m}-c_{j m} c_{l k} .
$$

The normalized determinant is cutoff $(\Lambda)$ independent. All dependence on $\Lambda$ comes from the zero temperature determinant, which up to a constant prefactor reads

$$
\Delta[\delta, T=0]=\exp \left[-i \sum_{1 \leq j \leq N_{t}} \Lambda \tau_{j} \frac{\left(\delta_{j}-\delta_{j-1}\right)}{2 \pi}\right] \prod_{1 \leq j<l \leq N_{t}}\left|\frac{\Lambda\left(\tau_{j}-\tau_{l}\right)}{\pi}\right|^{\left(\delta_{j}-\delta_{j-1}\right)\left(\delta_{l}-\delta_{l-1}\right) / 4 \pi^{2}},
$$

where we have defined the phases $\delta_{j} \equiv \delta\left(t_{j}+0\right)$. While a rigorous mathematical proof of these results is still missing,

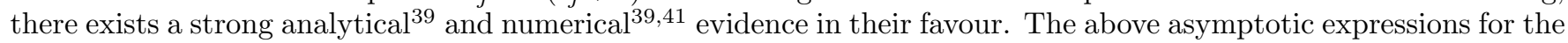
generalized Toeplitz determinants are used in Appendix B for evaluation of the Green's function in the nonequilibrium Luttinger liquid after the quench.

\section{Appendix B: Asymptotic behavior of the Green's function}

This appendix contains details of calculations of the asymptotic behavior of the determinants that lead to the results presented in SecIIIB.

Throughout the paper we use the nonequilibrium version of canonical bosonization developed in Refs. 37. 40 , In this framework nonequilibrium corrections to the right-movers' equal-time Green's function $G_{+}^{\gtrless}(\bar{t} ; \bar{x}, 0)$ [see Eq. 55$]$ are expressed in terms of Fredholm determinants

$$
\Delta_{\mu} \equiv \operatorname{Det}\left[\mathbb{1}+\left(e^{i \delta_{\mu}}-\mathbb{1}\right) f_{\mu}\right]
$$

with the counting phases $\delta_{\mu}[$ Eq. 10 ] $]$ which were found in section III A.

Let us consider first the equilibrium situation with distribution functions $f_{+}(\epsilon)=f_{-}(\epsilon)=f_{0}(\epsilon)=\theta(-\epsilon)$. Equations (A4) and $\mathrm{A} 6$ yield

$$
\begin{aligned}
& \Delta_{+}=G\left(1-s^{2}\right) G\left(1+s^{2}\right) G\left(1-c^{2}\right) G\left(1+c^{2}\right) e^{i \Lambda \bar{x} / v_{F}}\left|\frac{\Lambda \bar{x}}{\pi v_{F}}\right|^{-c^{4}-s^{4}}\left|\frac{(2 u \bar{t})^{2}-\bar{x}^{2}}{(2 u \bar{t})^{2}}\right|^{s^{2} c^{2}}, \\
& \Delta_{-}=G(1-c s)^{2} G(1+c s)^{2}\left|\frac{\Lambda \bar{x}}{\pi v_{F}}\right|^{-2 c^{2} s^{2}}\left|\frac{(2 u \bar{t})^{2}-\bar{x}^{2}}{(2 u \bar{t})^{2}}\right|^{s^{2} c^{2}} .
\end{aligned}
$$

The prefactors containing the Barnes G-functions $G$ do not directly follow from the asymptotic formulae for the generalized Toeplitz determinants. They can be found from the long-time limit where the factorization into simple Toeplitz determinants is applicable (we refer the reader to Ref. 38 for more details).

The Green's function following the equilibrium interaction quench is thus

$$
G_{+}^{<}(\bar{t} ; \bar{x}, 0) \propto e^{i \Lambda \bar{x} / v_{F}}\left|\frac{\Lambda \bar{x}}{\pi v_{F}}\right|^{-\left(c^{2}+s^{2}\right)^{2}}\left|\frac{(2 u \bar{t})^{2}-\bar{x}^{2}}{(2 u \bar{t})^{2}}\right|^{2 s^{2} c^{2}} .
$$

Since $2 c s=\tilde{\gamma}$ and $-\left(c^{2}+s^{2}\right)^{2}=-1-\tilde{\gamma}^{2}$ the power laws are in perfect agreement with the exact result (3) of Ref. 8 . In the following we will use the equilibrium quench as reference case to normalize our Fredholm determinants. 
The situation becomes more complicated when turning to nonequilibrium. We consider the double step distribution functions $f_{ \pm}(\epsilon)=(1-a) \theta(-\epsilon)+a \theta(U-\epsilon)$ for right- and left-movers as the initial steady state of the noninteracting Fermi sea before the quench. Using the asymptotic formulae $(\mathrm{A} 4)$ and $(\mathrm{A} 6)$ we obtain

$$
G_{+}^{<}(\bar{t} ; \bar{x}, 0)=G_{0+}^{<}(0, \bar{x}) \tilde{\Delta}_{+} \tilde{\Delta}_{-}
$$

for $|\bar{x}|,|2 u \bar{t}-| \bar{x}||,|2 u \bar{t}| \gg v_{F} / U$ with equilibrium-normalized determinants

$$
\begin{aligned}
\tilde{\Delta}_{+}= & \sum_{n_{1}, n_{2}, n_{3} \in \mathbb{Z}} \tilde{\Gamma}_{+}\left(n_{1}, n_{2}, n_{3}\right) e^{i\left(\beta_{1}+\beta_{2}\right) U|\bar{x}| / v_{F}} e^{i\left(n_{1}+n_{2}-n_{3}\right) U|\bar{x}| / v_{F}} e^{i n_{3} 2 U \bar{t} u / v_{F}} \\
& \times\left|\frac{\Lambda}{\pi v_{F}}\right|^{1+\gamma_{+}+V_{+}}|\bar{x}|^{1+X_{+}}(2 u \bar{t}-|\bar{x}|)^{D_{+}}(2 u \bar{t}+|\bar{x}|)^{S_{+}}(2 u \bar{t})^{T_{+}}\left|\frac{\pi U}{\Lambda}\right|^{V_{+}}, \\
\tilde{\Delta}_{-}= & \sum_{n_{4}, n_{5}, n_{6} \in \mathbb{Z}} \tilde{\Gamma}_{-}\left(n_{4}, n_{5}, n_{6}\right) e^{i\left(\beta_{3}+\beta_{4}\right) U|\bar{x}| / v_{F}} e^{i\left(n_{4}+n_{5}-n_{6}\right) U|\bar{x}| / v_{F}} e^{i n_{6} 2 U \bar{t} u / v_{F}} \\
& \times\left|\frac{\Lambda}{\pi v_{F}}\right|^{\gamma_{-}+V_{-}}|\bar{x}|^{X_{-}}(2 u \bar{t}-|\bar{x}|)^{D_{-}}(2 u \bar{t}+|\bar{x}|)^{S_{-}}(2 u \bar{t})^{T_{-}}\left|\frac{\pi U}{\Lambda}\right|^{V_{-}}
\end{aligned}
$$

for $2 u \bar{t}>\bar{x}>0$ and

$$
\begin{aligned}
\tilde{\Delta}_{+}= & \sum_{n_{1}, n_{2}, n_{3} \in \mathbb{Z}} \tilde{\Gamma}_{+}^{\prime}\left(n_{1}, n_{2}, n_{3}\right) e^{i\left(\beta_{1}+\beta_{2}\right) 2 U \bar{t} u / v_{F}} e^{i\left(n_{1}+n_{2}-n_{3}\right) U|\bar{x}| / v_{F}} e^{i n_{3} 2 U \bar{t} u / v_{F}} \\
& \times\left|\frac{\Lambda}{\pi v_{F}}\right|^{1+\gamma_{+}^{\prime}+V_{+}^{\prime}}|\bar{x}|^{1+X_{+}^{\prime}}(-2 u \bar{t}+|\bar{x}|)^{D_{+}^{\prime}}(2 u \bar{t}+|\bar{x}|)^{S_{+}^{\prime}}(2 u \bar{t})^{T_{+}^{\prime}}\left|\frac{\pi U}{\Lambda}\right|^{V_{+}^{\prime}}, \\
\tilde{\Delta}_{-}= & \sum_{n_{4}, n_{5}, n_{6} \in \mathbb{Z}} \tilde{\Gamma}_{-}^{\prime}\left(n_{4}, n_{5}, n_{6}\right) e^{i\left(\beta_{3}+\beta_{4}\right) 2 U \bar{t} u / v_{F}} e^{i\left(n_{4}+n_{5}-n_{6}\right) U|\bar{x}| / v_{F}} e^{i n_{6} 2 U \bar{t} u / v_{F}} \\
& \times\left|\frac{\Lambda}{\pi v_{F}}\right|^{\gamma_{-}^{\prime}+V_{-}^{\prime}}|\bar{x}|^{X_{-}^{\prime}}(-2 u \bar{t}+|\bar{x}|)^{D_{-}^{\prime}}(2 u \bar{t}+|\bar{x}|)^{S_{-}^{\prime}}(2 u \bar{t})^{T_{-}^{\prime}}\left|\frac{\pi U}{\Lambda}\right|^{V_{-}^{\prime}}
\end{aligned}
$$

for $0<2 u \bar{t}<\bar{x}$. Here, we left the $n_{j}$-dependence of the exponents $X_{ \pm}, T_{ \pm}, \ldots$ implicit. $\tilde{\Gamma}_{ \pm}, \tilde{\Gamma}_{ \pm}^{\prime}$ are numerical prefactors which are not known in general. The determinants for $\bar{x}<0$ are obtained by complex conjugation.

The exponents differ in the two regimes $2 u \bar{t} \gtrless|\bar{x}|$, which we consider below separately.

\section{Regime of separated phase pulses, $2 u \bar{t}>|\bar{x}|$}

Here the exponents are

$$
\begin{aligned}
X_{+}= & \left(-\beta_{2}+c^{2}-n_{2}\right)\left(\beta_{2}-c^{2}+n_{2}-n_{3}\right)+\left(-\beta_{1}-n_{1}+n_{3}\right)\left(\beta_{1}+n_{1}\right) \\
& +\left(-\beta_{2}-n_{2}+n_{3}\right)\left(\beta_{2}+n_{2}\right)+\left(-\beta_{1}-n_{1}-s^{2}\right)\left(\beta_{1}+n_{1}-n_{3}+s^{2}\right), \\
T_{+}= & \left(-n_{1}+n_{3}-s^{2}-\beta_{1}\right)\left(c^{2}-n_{2}-\beta_{2}\right)+\left(-n_{1}-s^{2}-\beta_{1}\right)\left(c^{2}-n_{2}+n_{3}-\beta_{2}\right) \\
& +\left(n_{1}-n_{3}+\beta_{1}\right)\left(n_{2}+\beta_{2}\right)+\left(n_{1}+\beta_{1}\right)\left(n_{2}-n_{3}+\beta_{2}\right), \\
D_{+}= & \left(n_{1}-n_{3}+\beta_{1}\right)\left(-n_{2}+n_{3}-\beta_{2}\right)+\left(-n_{1}+n_{3}-s^{2}-\beta_{1}\right)\left(-c^{2}+n_{2}-n_{3}+\beta_{2}\right), \\
S_{+}= & \left(\beta_{1}+n_{1}\right)\left(c^{2}-2\left(\beta_{2}+n_{2}\right)\right)-s^{2}\left(\beta_{2}-c^{2}+n_{2}\right), \\
\gamma_{+}= & -c^{4}-s^{4}, \\
X_{-}= & \left(-n_{4}+n_{6}-\beta_{3}\right)\left(n_{4}+\beta_{3}\right)+\left(-n_{4}+c s-\beta_{3}\right)\left(n_{4}-n_{6}-c s+\beta_{3}\right) \\
& +\left(-n_{5}+n_{6}-\beta_{4}\right)\left(n_{5}+\beta_{4}\right)+\left(-n_{5}-c s-\beta_{4}\right)\left(n_{5}-n_{6}+c s+\beta_{4}\right), \\
T_{-}= & \left(-n_{4}+n_{6}+c s-\beta_{3}\right)\left(-n_{5}-c s-\beta_{4}\right)+\left(-n_{4}+c s-\beta_{3}\right)\left(-n_{5}+n_{6}-c s-\beta_{4}\right) \\
& +\left(n_{4}-n_{6}+\beta_{3}\right)\left(n_{5}+\beta_{4}\right)+\left(n_{4}+\beta_{3}\right)\left(n_{5}-n_{6}+\beta_{4}\right), \\
D_{-}= & \left(n_{4}-n_{6}+\beta_{3}\right)\left(-n_{5}+n_{6}-\beta_{4}\right)+\left(-n_{4}+n_{6}+c s-\beta_{3}\right)\left(n_{5}-n_{6}+c s+\beta_{4}\right), \\
S_{-}= & c s\left(n_{5}+c s+\beta_{4}\right)+\left(n_{4}+\beta_{3}\right)\left(-c s-2\left(n_{5}+\beta_{4}\right)\right), \\
\gamma_{-}= & -2 c^{2} s^{2} .
\end{aligned}
$$


In the long-time limit $2 u \bar{t} \gg|\bar{x}|$, the powers simplify to $(2 u \bar{t}-|\bar{x}|)^{D_{ \pm}}(2 u \bar{t}+|\bar{x}|)^{S_{ \pm}}(2 u \bar{t})^{T_{ \pm}} \rightarrow(2 u \bar{t})^{\tilde{T}_{ \pm}}$with $\tilde{T}_{+}=$ $-2 n_{3}^{2} \leq 0$ and $\tilde{T}_{-}=-2 n_{6}^{2} \leq 0$. Thus the correlation function relaxes to a stationary solution where solely terms with $n_{3}=0=n_{6}$ contribute. The remaining powers simplify to

$$
\begin{aligned}
& X_{+}=-2\left(n_{1}-\frac{-s^{2}-2 \beta_{1}}{2}\right)^{2}-2\left(n_{2}-\frac{c^{2}-2 \beta_{2}}{2}\right)^{2}-\frac{c^{4}+s^{4}}{2}, \\
& V_{+}=-2\left(n_{1}-\frac{-s^{2}-2 \beta_{1}}{2}\right)^{2}-2\left(n_{2}-\frac{c^{2}-2 \beta_{2}}{2}\right)^{2}+\frac{c^{4}+s^{4}}{2}, \\
& X_{-}=-2\left(n_{4}-\frac{c s-2 \beta_{3}}{2}\right)^{2}-2\left(n_{5}-\frac{-c s-2 \beta_{4}}{2}\right)^{2}-c^{2} s^{2}, \\
& V_{-}=-2\left(n_{4}-\frac{c s-2 \beta_{3}}{2}\right)^{2}-2\left(n_{5}-\frac{-c s-2 \beta_{4}}{2}\right)^{2}+c^{2} s^{2} .
\end{aligned}
$$

Since in the long-time limit the phases split into independent pulses, all Fredholm determinants factorize into Toeplitz determinants and the prefactors can be found in the closed analytical form using the generalized Fisher-Hartwig formula 38

$$
\begin{aligned}
\tilde{\Gamma}_{+}\left(n_{1}, n_{2}, n_{3}=0\right)= & \frac{G\left(1-s^{2}-\beta_{1}-n_{1}\right) G\left(1+s^{2}+\beta_{1}+n_{1}\right) G\left(1+\beta_{1}+n_{1}\right) G\left(1-\beta_{1}-n_{1}\right)}{G\left(1-s^{2}\right) G\left(1+s^{2}\right)} \\
& \times \frac{G\left(1+c^{2}-\beta_{2}-n_{2}\right) G\left(1-c^{2}+\beta_{2}+n_{2}\right) G\left(1+\beta_{2}+n_{2}\right) G\left(1-\beta_{2}-n_{2}\right)}{G\left(1+c^{2}\right) G\left(1-c^{2}\right)}, \\
\tilde{\Gamma}_{-}\left(n_{4}, n_{5}, n_{6}=0\right)= & \frac{G\left(1+c s-\beta_{3}-n_{4}\right) G\left(1-c s+\beta_{3}+n_{4}\right) G\left(1+\beta_{3}+n_{4}\right) G\left(1-\beta_{3}-n_{4}\right)}{G(1+c s) G(1-c s)} \\
& \times \frac{G\left(1-c s-\beta_{4}-n_{5}\right) G\left(1+c s+\beta_{4}+n_{5}\right) G\left(1+\beta_{4}+n_{5}\right) G\left(1-\beta_{4}-n_{5}\right)}{G(1-c s) G(1+c s)} .
\end{aligned}
$$

For moderate repulsive interaction $\sqrt{2}-1 \leq K \leq 1$, the dominant powers $\operatorname{Re} X_{ \pm}$are due to $\left(n_{1}, n_{2}\right)=(0,1),(0,0)$ and $\left(n_{4}, n_{5}\right)=(0,0)$. These contributions are taken into account in 16 with $\tilde{\Gamma}_{1} \equiv \tilde{\Gamma}_{+}(0,0,0) \tilde{\Gamma}_{-}(0,0,0), \tilde{\Gamma}_{2} \equiv$ $\tilde{\Gamma}_{+}(0,1,0) \tilde{\Gamma}_{-}(0,0,0)$. In the equilibrium limit, $a \rightarrow 0$, prefactors vanish for all $n_{j}$ but $n_{1}=n_{2}=n_{4}=n_{5}=0$ for which one recovers the equilibrium exponents.

\section{Regime of overlapping phase pulses, $2 u \bar{t}<|\bar{x}|$}

Here the exponents are

$$
\begin{aligned}
X_{+}^{\prime}= & \left(-c^{2}+n_{2}-n_{3}-\beta_{1}\right)\left(c^{2}-n_{2}-\beta_{2}\right)+\left(-n_{1}-s^{2}-\beta_{1}\right)\left(n_{1}-n_{3}+s^{2}-\beta_{2}\right) \\
& +\left(-n_{2}+n_{3}+\beta_{1}\right)\left(n_{2}+\beta_{2}\right)+\left(n_{1}+\beta_{1}\right)\left(-n_{1}+n_{3}+\beta_{2}\right), \\
T_{+}^{\prime}= & \left(n_{2}-n_{3}-\beta_{1}\right)\left(n_{1}+\beta_{1}\right)+\left(-n_{1}-s^{2}-\beta_{1}\right)\left(c^{2}-n_{2}+n_{3}+\beta_{1}\right) \\
& +\left(n_{1}-n_{3}-\beta_{2}\right)\left(n_{2}+\beta_{2}\right)+\left(c^{2}-n_{2}-\beta_{2}\right)\left(-n_{1}+n_{3}-s^{2}+\beta_{2}\right), \\
D_{+}^{\prime}= & \left(-n_{2}+n_{3}+\beta_{1}\right)\left(n_{1}-n_{3}-\beta_{2}\right)+\left(-c^{2}+n_{2}-n_{3}-\beta_{1}\right)\left(-n_{1}+n_{3}-s^{2}+\beta_{2}\right), \\
S_{+}^{\prime}= & -s^{2}\left(-c^{2}+n_{2}+\beta_{2}\right)+\left(n_{1}+\beta_{1}\right)\left(c^{2}-2\left(n_{2}+\beta_{2}\right)\right), \\
X_{-}^{\prime}= & \left(n_{5}-n_{6}-c s-\beta_{3}\right)\left(-n_{5}+c s-\beta_{4}\right)+\left(-n_{4}-c s-\beta_{3}\right)\left(n_{4}-n_{6}+c s-\beta_{4}\right) \\
& +\left(-n_{5}+n_{6}+\beta_{3}\right)\left(n_{5}+\beta_{4}\right)+\left(n_{4}+\beta_{3}\right)\left(-n_{4}+n_{6}+\beta_{4}\right), \\
T_{-}^{\prime}= & \left(n_{5}-n_{6}-\beta_{3}\right)\left(n_{4}+\beta_{3}\right)+\left(-n_{4}-c s-\beta_{3}\right)\left(-n_{5}+n_{6}+c s+\beta_{3}\right) \\
& +\left(n_{4}-n_{6}-\beta_{4}\right)\left(n_{5}+\beta_{4}\right)+\left(-n_{5}+c s-\beta_{4}\right)\left(-n_{4}+n_{6}-c s+\beta_{4}\right), \\
D_{-}^{\prime}= & \left(-n_{5}+n_{6}+\beta_{3}\right)\left(n_{4}-n_{6}-\beta_{4}\right)+\left(n_{5}-n_{6}-c s-\beta_{3}\right)\left(-n_{4}+n_{6}-c s+\beta_{4}\right), \\
S_{-}^{\prime}= & -c s\left(n_{5}-c s+\beta_{4}\right)+\left(n_{4}+\beta_{3}\right)\left(c s-2\left(n_{5}+\beta_{4}\right)\right) .
\end{aligned}
$$

For long distances $|\bar{x}| \gg 2 u \bar{t}$ the power-law dependence on distance simplifies to $|\bar{x}|^{X_{ \pm}^{\prime}}(-2 u \bar{t}+|\bar{x}|)^{D_{ \pm}^{\prime}}(2 u \bar{t}+|\bar{x}|)^{S_{ \pm}^{\prime}} \rightarrow|\bar{x}|^{\tilde{X}_{ \pm}}$with the exponents

$$
\tilde{X}_{+}=-2\left(n_{3}+1 / 2-n_{1}-n_{2}\right)^{2}-\frac{1}{2}, \quad \tilde{X}_{-}=-2\left(n_{6}-n_{4}-n_{5}\right)^{2} .
$$


For $|\bar{x}| \rightarrow \infty$ all terms vanish except for $n_{3}=n_{1}+n_{2}$ or $n_{3}=n_{1}+n_{2}-1$, and $n_{6}=n_{4}+n_{5}$. Then $1+\tilde{X}_{+}=0=\tilde{X}_{-}$, i.e. the normalized determinants $\tilde{\Delta}_{ \pm}$are independent of $\bar{x}$, and correlations drop off like $G_{+}^{<}(\bar{t} ; \bar{x}, 0) \sim G_{0+}^{<}(\bar{t} ; \bar{x}, 0) \sim \bar{x}$.

The remaining exponents are

$$
T_{+}^{\prime}\left(V_{+}^{\prime}\right)=-2\left(n_{1}-\frac{-1 / 2-s^{2}-2 \beta_{1}}{2}\right)^{2}-2\left(n_{2}-\frac{-1 / 2+c^{2}-2 \beta_{2}}{2}\right)^{2}+\frac{1}{4} \mp c^{2} s^{2}
$$

for $n_{3}=n_{1}+n_{2}$,

$$
T_{+}^{\prime}\left(V_{+}^{\prime}\right)=-2\left(n_{1}-\frac{1 / 2-s^{2}-2 \beta_{1}}{2}\right)^{2}-2\left(n_{2}-\frac{1 / 2+c^{2}-2 \beta_{2}}{2}\right)^{2}+\frac{1}{4} \mp c^{2} s^{2}
$$

for $n_{3}=n_{1}+n_{2}-1$ and

$$
T_{-}^{\prime}\left(V_{-}^{\prime}\right)=-2\left(n_{4}-\frac{-c s-2 \beta_{3}}{2}\right)^{2}-2\left(n_{5}-\frac{c s-2 \beta_{4}}{2}\right)^{2} \mp c^{2} s^{2}
$$

for $n_{6}=n_{4}+n_{5}$.

1 T. Giamarchi, Quantum Physics in One Dimension (Oxford University Press, 2004).

${ }^{2}$ I. Bloch, J. Dalibard, and W. Zwerger, Rev. Mod. Phys. 80, 885 (2008).

3 A. Polkovnikov, K. Sengupta, A. Silva, M. Vengalattore, Rev. Mod. Phys. 83, 863 (2011).

${ }^{4}$ M. Greiner, O. Mandel, T. Haensch, and I. Bloch, Nature 419, 51 (2002).

5 T. Kinoshita, T. Wenger, and D. S. Weiss, Nature (London) 440, 900 (2006).

6 S. Hofferberth, I. Lesanovsky, B. Fischer, T. Schumm, and J. Schmiedmayer, Nature (London) 449, 324 (2007).

7 M. Gring, M. Kuhnert, T. Langen, T. Kitagawa, B. Rauer, M. Schreitl, I. Mazets, D. A. Smith, E. Demler, and J. Schmiedmayer, Science 337, 1318 (2012).

8 M. A. Cazalilla, Phys. Rev. Lett. 97, 156403 (2006).

9 M. Rigol, V. Dunjko, V. Yurovsky, and M. Olshanii, Phys. Rev. Lett. 98, 050405 (2007).

10 P. Calabrese and J. Cardy, Phys. Rev. Lett. 96, 136801 (2006); Journal of Statistical Mechanics: Theory and Experiment, P06008 (2007); ibid, P10004 (2007).

11 C. Kollath, A. M. Läuchli, and E. Altman, Phys. Rev. Lett. 98, 180601 (2007); A. M. Läuchli and C. Kollath, Journal of Statistical Mechanics: Theory and Experiment, P05018 (2008).

12 D. M. Gangardt and M. Pustilnik, Phys. Rev. A 77, 041604(R) (2008).

13 M. Cramer, A. Flesch, I. P. McCulloch, U. Schollwöck, and J. Eisert, Phys. Rev. Lett. 101, 063001 (2008).

14 A. Iucci and M. A. Cazalilla, Phys. Rev. A 80, 063619 (2009).

15 M. Rigol, Phys. Rev. A 80, 053607 (2009).

16 G. S. Uhrig, Phys. Rev. A 80, 061602 (2009); S. A. Hamerla and G. S. Uhrig, New Journal of Physics 15, 073012 (2013).

17 S. R. Manmana, S. Wessel, R. M. Noack, and A. Muramatsu, Phys. Rev. B 79, 155104 (2009).

18 J. Sabio and S. Kehrein, New J. Phys. 12, 055008 (2010).
19 P. Barmettler, M. Punk, V. Gritsev, E. Demler, and E. Altman, New Journal of Physics 12, 055017 (2010).

20 A. Mitra and T. Giamarchi, Phys. Rev. Lett. 107, 150602 (2011); J. Lancaster and T. Giamarchi, and A. Mitra, Phys. Rev. B 84, 075143 (2011); A. Mitra, Phys. Rev. Lett. 109, 260601 (2012); A. Mitra, Phys. Rev. B 87, 205109 (2013).

21 M. S. Foster, T. C. Berkelbach, D. R. Reichman, and E. A. Yuzbashyan, Phys. Rev. B 84, 085146 (2011).

22 E. Perfetto and G. Stefanucci, Europhys. Lett. 95, 10006 (2011).

23 B. Dora, M. Haque, and G. Zarand, Phys. Rev. Lett. 106, 156406 (2011); B. Dora, A. Bacsi, and G. Zarand, Phys. Rev. B 86, 161109 (2012). Á Bácsi, B. Dóra, arXiv:1307.7582 (2013)

24 C. Karrasch, J. Rentrop, D. Schuricht, V. Meden, Phys. Rev. Lett. 109, 126406 (2012); J. Rentrop, D. Schuricht, and V. Meden, New Journal of Physics 14, 075001 (2012).

25 P. Calabrese, F. H.L. Essler, M. Fagotti, Phys. Rev. Lett. 106, 227203 (2011); J. Stat. Mech., P07016 (2012); ibid P07022 (2012).

26 N. Nessi, A. Iucci, Phys. Rev. B 87, 085137 (2013)

27 Y.-Fu Chen et al., Phys. Rev. Lett. 102, 036804 (2009).

28 C. Altimiras, H. le Sueur, U. Gennser, et al., Nature Physics, 634 (2010); H. le Sueur, C. Altimiras, U. Gennser, et al., Phys. Rev. Lett. 105056803 (2010); H. le Sueur, C. Altimiras, U. Gennser, et al., Phys. Rev. Lett. 105 226804 (2010).

29 D. T. McClure et al., Phys. Rev. Lett. 103, 206806 (2009).

30 Y. Yamauchi et al., Phys. Rev. B 79, 161306(R) (2009).

31 Yiming Zhang et al., Phys. Rev. B 79, 241304(R) (2009).

32 N. Ofek et al., Proc. Natl. Acad. Sci. USA 107, 5276 (2010).

33 Y. Ji, Y. C. Chung, D. Sprinzak, M. Heiblum, D. Mahalu, and H. Shtrikman, Nature (London) 422, 415 (2003); I. Neder, M. Heiblum, Y. Levinson, D. Mahalu, and V. Umansky, Phys. Rev. Lett. 96, 016804 (2006); I. Neder, M. 
Heiblum, D. Mahalu, and V. Umansky, Phys. Rev. Lett. 98, 036803 (2007); I. Neder, F. Marquardt, M. Heiblum, D. Mahalu, and V. Umansky, Nat. Phys. 3, 534 (2007)

34 P. Roulleau, F. Portier, D. C. Glattli, P. Roche, A. Cavanna, G. Faini, U. Gennser, and D. Mailly, Phys. Rev. B 76, 161309(R)(2007); P. Roulleau, F. Portier, D. C. Glattli, P. Roche, A. Cavanna, G. Faini, U. Gennser, and D. Mailly, Phys. Rev. Lett. 100, 126802(2008); P. Roulleau, F. Portier, P. Roche, A. Cavanna, G. Faini, U. Gennser, and D. Mailly, Phys. Rev. Lett. 101, 186803 (2008); ibid 102, 236802 (2009); P-A. Huynh, F. Portier, H. le Sueur, G. Faini, U. Gennser, D. Mailly, F. Pierre, W. Wegscheider, P. Roche, Phys. Rev. Lett. 108, 256802 (2012).

${ }^{35}$ L. V. Litvin, H.-P. Tranitz, W. Wegscheider, and C. Strunk, Phys. Rev. B 75, 033315 (2007); L. V. Litvin, A. Helzel, H.-P. Tranitz, W. Wegscheider, and C. Strunk, Phys. Rev. B 78, 075303 (2008); L. V. Litvin, A. Helzel, H.-P. Tranitz, W. Wegscheider, and C. Strunk, Phys. Rev. B 81, 205425 (2010).

36 E. Bieri, M. Weiss, O. Göktas, M. Hauser, C. Schönenberger, and S. Oberholzer, Phys. Rev. B 79, 245324 (2009).
37 D. B. Gutman, Y. Gefen, and A. D. Mirlin, Europhys. Letters 90, 37003 (2010); Phys. Rev. B 81, 085436 (2010).

38 D. B. Gutman, Y. Gefen, and A. D. Mirlin, J. Phys. A: Math. Theor. 44, 165003 (2011).

39 I. V. Protopopov, D. B. Gutman, and A. D. Mirlin, Lith. J. Phys. 52, 165 (2012).

40 I. V. Protopopov, D. B. Gutman, A. D. Mirlin, Phys. Rev. Lett. 110, 216404 (2013).

41 S. Ngo Dinh, D. A. Bagrets, and A. D. Mirlin, Phys. Rev. B 87, 195433 (2013).

42 D. L. Kovrizhin and J. T. Chalker, Phys. Rev. B 80, 161306(R) (2009); M. J. Rufino, D. L. Kovrizhin, and J. T. Chalker, Phys. Rev. B 87, 045120 (2013).

43 I. P. Levkivskyi and E. V. Sukhorukov, Phys. Rev. Lett. 103, 036801 (2009).

44 D. L. Kovrizhin, J. T. Chalker, Phys. Rev. B 84, 085105 (2011); Phys. Rev. Lett. 109, 106403 (2012).

45 I. P. Levkivskyi and E. V. Sukhorukov, Phys. Rev. B 85, 075309 (2012).

46 P. Deift, A. Its, and I. Krasovsky, Ann. Math. 174-2, 1243 (2011). 